\title{
Nacimiento de las Escuelas Normales gaditanas: un análisis de las matrículas que evidencian su repercusión
}

\author{
Carmen Vázquez Domínguez \\ Profesora del Área de Teoría e Historia de la Educación de la Universidad de Cádiz \\ carmen.dominguez@uca.es \\ ORCID: https://orcid.org/0000-0002-7923-8243
}

RESUMEN: Este trabajo tiene como finalidad el estudio del establecimiento de centros específicos de formación del profesorado, las Escuelas Normales, en Cádiz. Paralelamente, además, se pretende contribuir mediante el análisis de su matrícula a evidenciar lo que supusieron estos centros para las inquietudes femeninas de instrucción.

Para su elaboración hemos recurrimos a la metodología habitualmente utilizada en la investigación histórico-educativa, yendo a las fuentes primarias del fondo documental del Archivo de la Universidad de Cádiz (AUCA).

Palabras clave: Escuelas Normales, Educación Femenina, Matrícula.

The origin of the Normal Schools in Cadiz: an analysis of the enrollment showing their repercussion

ABSTRACT: This work has the purpose of studying the establishment of specific teacher training centers, the Normal Schools, in Cádiz. At the same time, it is also intending to contribute through the analysis of the Schools' enrollment to show what these centers meant for the feminine concerns of instruction.

For its development, it has been resorted to the methodology usually used in the historical-educational research, going to the primary sources of the documentary collection of the Archive of the University of Cádiz.

Keywords: Normal Schools, Women Education, Enrollment. 


\section{A MODO DE INTRODUCCIÓN: EL FONDO DOCUMENTAL SOBRE LAS ESCUELAS NORMALES DE} CÁDIZ

En tiempos de la creación de las Escuelas Normales, como sabemos, los centros de educación superior de la ciudad dependían del distrito de la Universidad de Sevilla, al que había que enviar la documentación oficial que se requiriera; entendemos que es por ello, que las direcciones de las Escuelas de Magisterio gaditanas, y suponemos que también las de las demás provincias, salvaguardaban toda la documentación oficial, lo que en el caso de Cádiz permite reconstruir la historia de las Escuelas Normales, tanto la femenina como la masculina, sin necesidad de recurrir al Archivo Histórico de la Universidad de Sevilla. Hoy toda la documentación se encuentran en el archivo de nuestra universidad; es más, sin llegar a conocer el motivo, después de gestiones y consultas realizadas tenemos constancias que en la actualidad el archivo sevillano cuenta con un fondo limitado a dos legajos, el 515 de expediente (1875 a 1890) y el 662 de listas de alumnado (1858 a 1866), comunicaciones (1858-1868) y expedientes (1857-1865).

En sus orígenes, la documentación de los fondos históricos procedentes de la Escuela Normal hasta su constitución como Facultad de Ciencias de la Educación se encontraban físicamente archivada en dos salas destinadas a tal fin en el edificio de la citada facultad, en Puerto Real: una dedicada al siglo XIX y otra al XX. Posteriormente, en años relativamente recientes, se produjo su traslado al Archivo General de la Universidad de Cádiz como resultado de la decisión tomada por las autoridades universitarias competentes de centralizar toda la documentación.

A partir de la información obtenida de los documentos correspondientes a la creación y a los primeros años del funcionamiento como Escuela Normal de Magisterio, que abarcan desde 1857 hasta 1863, los libros oficiales de las entradas y las salidas de correspondencia, los estudios estadísticos de aprovechamiento, los expedientes del alumnado, etc., se ha podido obtener una visión bastante definida del establecimiento en su conjunto y, especialmente, en su sección destinada a la formación del magisterio femenino.

\section{APROXIMACIÓN A LA HISTORIA DEL MAGISTERIO EN ESPAÑA}

La preocupación por la preparación del magisterio en España se extiende a lo largo de un amplio período de tiempo que se puede dividir en tres etapas históricas. La primera etapa abarcaría desde 1370 hasta 1780 en la que se recogen las primeras disposiciones con respecto a los maestros dentro del marco gremial que es común al desempeño de su labor entonces; la segunda, hasta 1838-39, en la que se intenta proporcionar una formación adecuada a los maestros, a través del Colegio Académico de Primeras Letras, cuando todavía el Estado no ha asumido la responsabilidad de su preparación. La fecha de 1838-39, marca el primer intento a cargo de Pablo Montesino para que esto sea así con la instancia a fundar la primera Escuela Normal para el magisterio en Madrid; y, por último, la tercera etapa corresponde al período en el que las Escuelas Normales, que desde ahora se hacen cargo de la formación de los/as maestros/as, se expanden por todo el territorio nacional en virtud del mandato de la Ley de 
Claudio Moyano (1857) que contempla la creación de una en cada capital de provincia (Lorenzo 1995a, p. 205).

En la primera etapa histórica, la formación del maestro tiene un carácter gremial, es decir, es el resultado de permanecer al lado de otro maestro como aprendiz, y posteriormente, tras la realización de un examen, ya se podía ejercer como tal. El magisterio tenía una organización similar a la de cualquier otra profesión gremial. Su formación teórica era nula, pues todo el proceso de aprendizaje estaba unido a la práctica y era resultado de ella. Los docentes de este período no necesitaban tener más instrucción que la que impartían, es decir, únicamente debían dominar la lectura, la escritura, la caligrafía y el cálculo.

En 1370, por la Real Cédula de Enrique II se prohíbe el ejercicio del magisterio a todo aquel que no haya superado un examen donde se valora el conocimiento de la profesión, y se establece que sea desde ahora el Consejo de Castilla el encargado de la realización de la citada prueba así como de expedir el título correspondiente. Para superar la prueba, es obligatorio el cumplimiento de tres requerimientos: la limpieza de sangre, la demostración de una recta conducta de vida y la realización de un examen sobre formación cristiana. En años posteriores, esta cédula será ratificada en tiempos de los Reyes Católicos, de Carlos I, Felipe II y Felipe III.

En 1642, durante el reinado de Felipe IV y con la autorización de este, se reúnen todos los maestros de Madrid con la intención de mejorar la enseñanza. Se crea la Hermandad de San Casiano (Ventajas 2007, p. 48), a la que, con el reconocimiento del Consejo de Castilla, se le concede la autoridad para examinar a todos los maestros del reino.

A lo largo de todo el siglo XVIII, nos encontramos con el intento de regular y controlar la función docente. Se modernizará la Hermandad de San Casiano, que según Capitán (1991, p. 819) es reemplazada en 1780 por la creación del Colegio Académico de Maestros de Primeras Letras $^{1}$. Mientras que la primera atiende fundamentalmente los intereses de sus integrantes, el segundo se plantea como objetivo prioritario el desarrollo de la educación popular y vela por ella promoviendo buenos rendimientos en la fe católica, en el cálculo, la lectura y la escritura, y en el desarrollo de virtudes para la buena actuación de la infancia (Lorenzo 1995b, p. 213). Desde esta fecha es cuando podemos empezar a hablar de una verdadera preocupación por la formación teórica del profesorado.

Con la desaparición de la Hermandad, las posteriores instituciones pierden las facultades de examinar a los maestros y de inspeccionar las escuelas, que pasarán a ser competencia de la Junta General de Exámenes y, con posterioridad, de la Secretaría de Gracia y Justicia, la Dirección General de Estudios y la Junta Superior de Inspección (Lorenzo 1995c, p. 213). Un paso muy importante para ir garantizando el control estatal sobre dicha profesión.

A principios del siglo XIX, se crean la Real Junta y las Juntas de capital de provincia con el objeto de examinar a los maestros. Es a partir de 1824, durante el reinado de Fernando VII, cuando se realizan una serie de cambios que terminan por asignar esta función a la Junta

\footnotetext{
1 “El Colegio Académico del Noble Arte de Primeras Letras (1780) se establecía, por Real Provisión de 22 de diciembre, dirigido al adelantamiento y mayor perfección de las Artes de Primeras Letras..."
} 
Suprema de Caridad; no obstante, un año más tarde, el Plan Calomarde traspasa esta competencia a la Junta Superior de Inspección de todas las Escuelas del Reino y a las Juntas de capital de provincia (Melcón 1992, p. 95).

Con posterioridad a la muerte de Fernando VII, a partir de las regencias del reinado de Isabel II y tras la vuelta de los exiliados liberales, se inicia un período marcado por un desarrollo legislativo escolar. En 1833 se crea el Ministerio de Fomento, en el que se asientan los principios de las reformas liberales; en 1836 son aprobados el Plan General de Instrucción Pública y el Reglamento de Instrucción Primaria, y también aparecen las Juntas Provinciales, dependientes del gobierno central a través de la Dirección General de Educación.

En este contexto surgen las Escuelas Normales en España, que, según Guzmán (1986, p. 36), lo hacen como consecuencia de la mezcla de tres necesidades: la primera responde al paulatino incremento de la importancia que la sociedad concede a la formación primaria como garantía de progreso; la segunda, la de preparar en centros adecuados al personal que se encargue de la docencia en este nivel de enseñanza; y, por último, la más concreta es la necesidad de contar con docentes que sepan leer y escribir, de acuerdo con lo que van a enseñar, y con los recursos metodológicos adecuados para ello.

\subsection{La formación de la maestra}

Históricamente, muchos de los trabajos y actividades femeninos no obligaban a establecer una formación institucionalizada y sistemática, por lo que la preparación se adquiría a través de la práctica, y ello explica la falta general de interés en su educación. Según Scanlon (1987, p. 194) es a partir de la Ley Moyano cuando se produce un avance realmente significativo para la educación femenina con respecto a las legislaciones precedentes, pues, en ella, concretamente en el artículo 100, se establece como obligatoria la creación de escuelas de niñas en pueblos que tengan más de 500 habitantes y, con ello, se reconoce por primera vez el derecho de instrucción de las niñas. Para esta misma autora, esta medida es determinante para la aparición de instituciones específicas que se encarguen de la preparación del personal que atenderá estas escuelas; es por ello por lo que la misma ley, en el artículo 114, propone la creación de Escuelas Normales Femeninas, aunque no serán obligatorias en todas las provincias. De tal circunstancia se desprende que la formación de las maestras será limitada y diferente, pues el énfasis se pondrá en las materias domésticas, ámbito hacia el que se encamina a la mujer.

Capel (1986, p. 424) señala que, durante la segunda mitad del siglo XIX y hasta bien entrado el siguiente siglo, las escuelas de magisterio son los mayores centros de adquisición de cultura para las mujeres y los únicos estudios de nivel no elemental en los que las mujeres son aceptadas sin reservas.

La Escuela Central de Madrid abre sus puertas en $1858^{2}$ y podemos considerarla el primer establecimiento con aspiraciones más amplias, pues, aunque se concibe para preparar a las maestras, también es, en principio, un centro piloto que pretende marcar las directrices y

\footnotetext{
${ }^{2}$ R. O. de 24 de febrero de 1858.
} 
extenderse como modelo al resto del territorio nacional. La Junta de Damas de Honor y Mérito será la encargada de su dirección y guarda, y abarcará su programa de estudios, principios y métodos de educación además de estar acorde con el de la escuela elemental y superior de niñas; su enfoque debe ser teórico y práctico, lo que lleva a agregarle una escuela de enseñanza mutua lancasteriana. Las alumnas de este centro serán externas (Pérez 1990, p. 35).

No queremos decir que con anterioridad no existan centros provinciales de formación para las aspirantes a maestras promovidos por alguna persona, algún grupo o alguna institución de la localidad.

\subsection{Las Escuelas Normales de Cádiz}

La preparación de los futuros docentes en las Escuelas Normales en España y la obligatoriedad de estar en posesión de un título para el ejercicio del magisterio van produciendo cambios encaminados a la obtención de un personal cualificado para atender las escuelas de instrucción primaria, tanto de un sexo como del otro.

Las Escuelas Normales de Cádiz, tanto la femenina como la masculina, abren sus puertas, según la documentación del Archivo de la Universidad de Cádiz (AUCA), en el año 1857 en el número 10 de la calle Bulas, en la actual Torre Tavira, alquilando a la Diputación Provincial el local. Ambas comienzan a funcionar a la par y se encuentran unidas no solo físicamente, al compartir el mismo edificio, sino también a nivel administrativo. Por esta razón, en esta primera etapa evolucionan conjuntamente e incluso comparten profesorado, puesto que en sus comienzos algunos docentes imparten clases en los dos centros.

Imagen 1. Oficio creación de las Escuelas Normales de Maestras, 1857

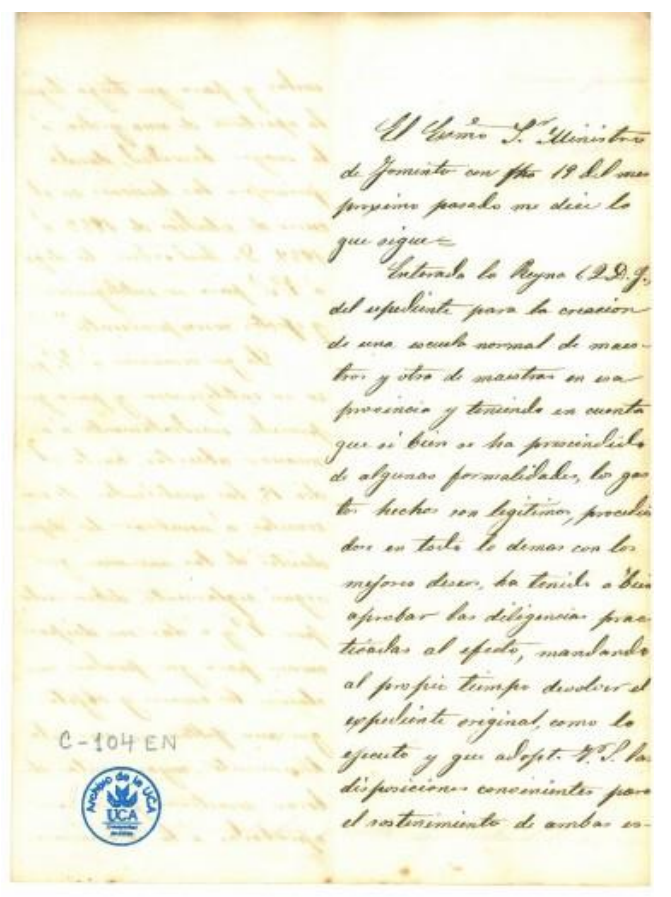

Fuente: Archivo de la Universidad de Cádiz (AUCA). C-104 EN

DOI del artículo:

http://dx.doi.org/10.25267/Cuad investig fondos arch UCA.2019.i1.07 
La creación simultánea de las dos Escuelas es un hecho singular en la provincia de Cádiz, junto con el caso de Murcia y Guadalajara, puesto que las escuelas del resto de las provincias de España aparecen de una manera gradual. El artículo 114 de la Ley de Instrucción Pública de 9 de septiembre de 1857 establece que "[e]l Gobierno procurará que se establezcan Escuelas Normales de Maestras para mejorar la instrucción de las niñas”, es decir, sugiere, apoya, recomienda, pero en ningún caso obliga al inmediato cumplimiento de lo mencionado, al contrario de lo que ocurre con las Escuelas de Maestros, cuya creación, no obstante, también se dilata en el tiempo. El valor otorgado, hasta este momento histórico, a los estudios de los varones en detrimento de los de las niñas se ve reflejado legislativamente, pero también en la organización y en la práctica diaria del inicio de estas Escuelas (Vázquez 2010, p. 36).

Hasta el año 1869, ambas escuelas caminaban juntas, y la Normal masculina ejercía administrativamente la tutela de la femenina. Con el R.D. de 8 de diciembre de este mismo año, la Escuela Normal de maestras adquiere autonomía con respecto a la primera, y comienza un nuevo período para esta en el que contará con su propio claustro y su presupuesto.

En el año 1881, se describe la calidad del nuevo edificio. En su nueva ubicación de la calle Bulas, es la segunda planta la que se destina a la Escuela de Maestras.

“Inst $4^{a}$ El edificio que ocupa la Escuela Normal es propiedad de S. José Ma Uceda, quien la tiene alquilada á la Exma. Diputacion provincial en 5000 pest. anuales. Dicho edificio es una magnífica casa de buen aspecto y capacidad bastante, está situado en el centro de la población, y en paraje elevado, ventilado y sano. Los planos adjuntos de las plantas bajas y principal dan una idea exacta de las dimensiones y distribución de las clases y gabinete, claros y espaciosos salones cubiertos de cielos rasos. La Dirección, Secretaría, Biblioteca y habitaciones del Director se hallan en el entresuelo. La del conserje y mozo en la planta baja. "3

Aunque ya las escuelas vienen funcionando separadas administrativamente, en el año 1883 se lleva a término la separación real, pues, dando un paso más, se separarán físicamente, ubicándose en distintos edificios.

Sin embargo, entendemos que la inauguración del curso 57/58 no se realiza con normalidad, pues entonces el acondicionamiento de la finca para su nueva aventura docente no está terminado ${ }^{4}$. De nuevo, el mismo gobernador y presidente de la Comisión Superior de Instrucción Primaria se pone en contacto con el responsable de las Normales y, en comunicado de 14 de octubre, rechaza una prórroga del período de matriculación hasta finales

\footnotetext{
${ }^{3}$ Doc. entre otras cuestiones sobre las condiciones del edificio que ocupa las Escuelas Normales. C-167-27 EN del Archivo de la Universidad de Cádiz (AUCA).

${ }^{4}$ Carta de 3 de noviembre de 1857 de D. Joaquín Asencio al Director de las Escuelas, en la que se concede un anticipo de 12.000 r. destinados a las obras del establecimiento bajo la obligación por parte del Director de presentar las cuentas de la inversión, justificando las mismas. C-585 EN del Archivo de la Universidad de Cádiz (AUCA).
} 
de octubre, una solicitud que se ha tramitado desde los centros de magisterio, por lo que entendemos que hay un retraso en la matrícula del alumnado o bien la demanda es insuficiente hasta la fecha.

De acuerdo con lo que se establece por ley, se abre el período de matriculación, en que todo aspirante debe entregar en la Secretaría del centro lo siguiente: una fe de bautismo legalizada que acredite los 16 años cumplidos; un certificado de buena conducta, que debe estar expedido por el alcalde y el cura párroco de su domicilio; un certificado médico en el que conste que el aspirante o la aspirante no padece ninguna enfermedad contagiosa en el momento de la solicitud ni tiene un defecto corporal que le pueda inhabilitar para ejercer la futura docencia; y, finalmente, una autorización del padre o del tutor para poder cursar los estudios de Magisterio. Para la admisión, una vez presentada la documentación, se debe superar un examen sobre las materias que se imparten en la instrucción primaria elemental y abonar una tasa de 80 r., la mitad en el momento de la matrícula y el resto al finalizar el curso $^{5}$. Las maestras que puedan acreditar que tienen una escuela establecida en la provincia podrán acceder a cursar sus estudios en la Normal de forma gratuita, al igual que sucede con las aspirantes a entrar en el curso de ampliación y que tienen una escuela abierta, que deberán presentar una copia legal de su título y un certificado de buena conducta ${ }^{6}$.

En los primeros años de las Escuelas Normales, se debió de realizar desde la Administración central un seguimiento bastante directo de ellas, pues en el año siguiente a su implantación en la ciudad se solicitó información desde la dirección de la Escuela Normal Superior de Sevilla sobre su ubicación en Cádiz y su fecha de creación, su organización, su presupuesto, el estado de sus locales y los medios materiales de los que disponían, ya que se habían recibido peticiones relativas a estas cuestiones desde el Ministerio de Fomento con el objeto de conocer la situación de las Escuelas Normales de las provincias andaluzas ${ }^{7}$.

La importancia de las Escuelas queda sobradamente probada cuando se las designa como un lugar de visita oficial para la reina Isabel II en su viaje por Andalucía en otoño de $1862^{8}$. Por esta razón, en enero de 1858 se invita al director y al profesorado del centro a asistir, junto con el gobernador militar de la provincia, al acto de celebración con motivo del día de $\mathrm{S}$. A. R. el Sr. Príncipe de Asturias ${ }^{9}$. Ello le es comunicado al director un día antes de la visita,

${ }^{5}$ Doc. sobre requisitos en matrículas de las Escuelas Normales de Cádiz. C-167-28 EN del Archivo de la Universidad de Cádiz (AUCA).

${ }^{6}$ Doc. sobre requisitos en matrículas de las Escuelas Normales de Cádiz. C-167-28 EN del Archivo de la Universidad de Cádiz (AUCA) Iguales condiciones de excepcionalidad serán aplicadas en el caso de los aspirantes a maestros.

${ }^{7}$ Doc. de correspondencia oficial de 24 de agosto, 10 de octubre y 24 de noviembre de 1858 . C-104 EN del Archivo de la Universidad de Cádiz (AUCA).

${ }^{8}$ Este mismo año Isabel II había dado a luz y por este motivo se organizó en junio un Solemne Te Deum en la Catedral de Cádiz al que se invitan al Director y profesorado de las Escuelas Normales. C-104 EN del Archivo de la Universidad de Cádiz (AUCA).

${ }^{9}$ Doc. de correspondencia de 22 de enero de 1859, traslado de invitación a un acto oficial desde la Junta de Instrucción pública provincial C-104 EN del Archivo de la Universidad de Cádiz (AUCA) 
que se realizará a las doce de la mañana del 2 de octubre ${ }^{10}$. En esta misma línea de centro formativo prestigioso, existe una felicitación a la dirección de la Escuela Normal por parte de la Inspección en la que le pide que haga extensiva la enhorabuena a todo el profesorado por los resultados obtenidos por el alumnado, pues, tras los exámenes de fin de curso, se ha podido comprobar el nivel de conocimientos adquiridos y, según la propia inspección, son la mejor prueba del nivel de formación que se imparte en un centro ${ }^{11}$.

Las Escuelas Normales, en estos años, no solo se caracterizaron por preparar a futuros docentes. El atraso cultural de España, que ya venía de antiguo, se podía paliar gracias a la labor que en las provincias realizaban estos centros, pues funcionaban como núcleos culturales sin suponer un gasto excesivo para las arcas públicas ${ }^{12}$. En este sentido, las Escuelas Normales eran las garantes del nivel de instrucción elemental del personal que aspiraba a formarse en otros centros de capacitación profesional. Por ejemplo, en 1863 hay constancia de que el profesorado de las Normales se encarga de examinar a alumnas para comprobar que superan los conocimientos elementales necesarios para acceder a los estudios de matronas o parteras dependientes de la Facultad de Medicina de la ciudad ${ }^{13}$, según establece el Reglamento de 1861 sobre las enseñanzas de Practicantes y Matronas dependientes de la Universidad Literaria de Sevilla. Su reconocimiento académico se dio desde sus primeros momentos, pues en 1859 el recién creado Colegio de Farmacéuticos de la ciudad quiso establecer cooperación profesional con las Normales y su profesorado, y les trasladó sus Estatutos y Reglamentos en los que se recogen los beneficios de una eficaz colaboración entre ambas instituciones ${ }^{14}$.

${ }^{10}$ Documento de comunicación de visita de Isabel II a la Escuela Normal. C-104 EN del Archivo de la Universidad de Cádiz (AUCA).

11 Doc. de 3 de junio de 1862 en el que se comunica a D. Manuel M. Romero desde Inspección las felicitaciones por la labor formativa que se realiza desde la Escuelas Normales. Entendemos que incluye también a la Escuela femenina y a las alumnas, pues aunque se hable en masculino es una fecha en que ambas Escuelas se encuentran administrativamente unidas.

12 "El presupuesto de las escuelas normales es hoy mezquino e insuficiente en todos los conceptos. Profesorado en el cual hay directores con 2.500 pesetas de sueldo y profesores con $1750 \ldots .$. Escuelas con 250 pesetas para todos los gastos de material y entretenimiento del edificio, no pueden tener recursos necesario para dar en buenas condiciones la enseñanza, ni por lo que se refiere a los elementos indispensables en métodos y procedimientos,..... a pesar de todos los esfuerzos del personal docente." Cossío, M.B.: "Número de Escuelas Normales que debe haber en España”. En: Boletín Institución Libre de Enseñanza, núm. 326, 1890.

${ }^{13}$ Comunicación de 19 de enero de 1863 del Decano de la Facultad de Medicina al Director de las Escuelas Normales sobre verificación de exámenes de las parteras. C.104 de EN del Archivo universidad de Cádiz (AUCA).

${ }^{14}$ Doc. de correspondencia de 8 de febrero de 1859, C.104 de EN del Archivo de la Universidad de Cádiz (AUCA). Los Estatutos y el Reglamento del Colegio de Farmacéuticos habían sido creados por R. O. de 31 de julio de 1858 . 


\subsection{La matrícula de las Escuelas Normales gaditanas: su evolución en el siglo XIX}

Para obtener una visión general de la importancia de la Escuelas Normales en la ciudad se hace necesario proceder al análisis de cómo se realiza la matrícula en ambos centros. La siguiente tabla comparativa se ha elaborado seleccionando la matrícula de la Normal masculina y la de la femenina en intervalos de cinco años. Hemos decidido introducir en la gráfica los primeros años de su existencia, los cursos 1857/58 y 1858/59, para seguidamente, ya que no incluimos el curso 1859/60, pasar al curso 1860/61; consideramos que son lo suficientemente ilustrativos del volumen de alumnado que acogen estos centros en su arranque histórico.

Los datos usados en este apartado han sido seleccionados y confeccionados a partir de la consulta de los libros L-1 EN, L-10 EN y L-2 EN del fondo del Archivo Universidad de Cádiz (AUCA) y que se corresponden con los libros 1, 3 y 4 del archivo histórico de la Facultad de Ciencias de la Educación antes de su traslado. Se ha realizado un barrido de los registros del alumnado que consta matriculado en las Escuelas Normales durante el siglo XIX. Para la elaboración de las tablas, se han utilizado los datos relativos a la matrícula masculina proporcionados por las profesoras Plaza y Pascual (2000) en su libro sobre los archivos de las Escuelas Normales de Cádiz, ya que presentan un cómputo global de esta matrícula.

Sin embargo, con respecto a los registros de la matrícula femenina, se han analizado los reseñados en estos libros para, posteriormente, acudir a la publicación citada y contrastar los datos. Hemos de señalar que, exceptuando algunos apuntes en concreto, probablemente resultantes de las operaciones realizadas por las profesoras, además de las fuentes y otros documentos que fueron utilizados, estamos totalmente de acuerdo con las cifras que dan.

Los datos incluidos en el siguiente análisis se refieren a la matrícula del alumnado sin entrar en la posterior finalización de los estudios ni en la solicitud del título acreditativo, aspectos apuntados en otra documentación diferente a la utilizada. 
Tabla I. Evolución de la matrícula en las Escuelas Normales de Cádiz, 1857-1899

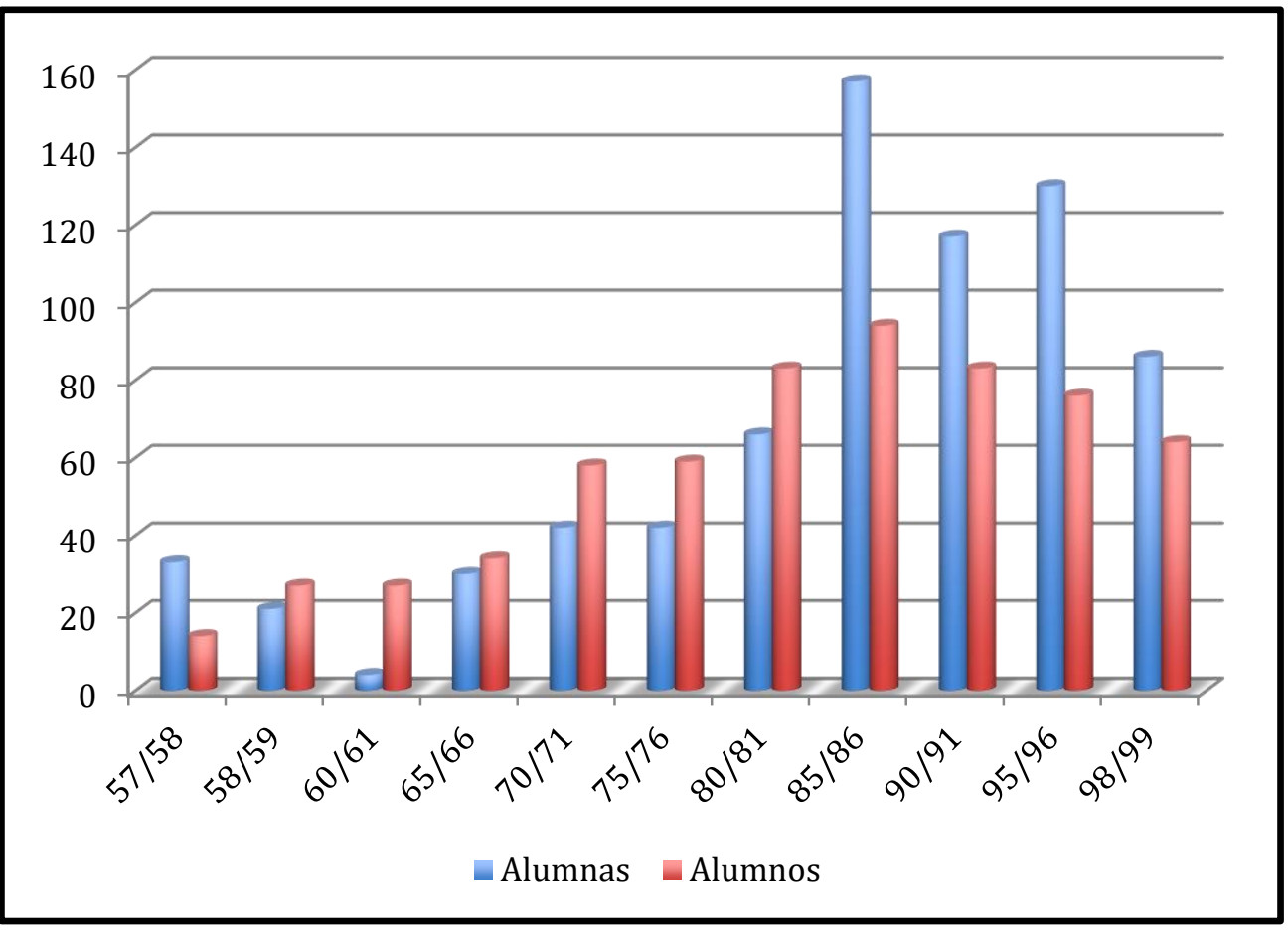

Fuente: Elaboración propia (2015)

Observamos que la matrícula en la Escuela Normal femenina alcanza casi el doble que la masculina en el curso 1857/58, situación que podría explicarse tanto por el ansia de formación de las jóvenes como por la escasez de instituciones encaminadas a la cualificación profesional de ellas. Estos centros nacen en la provincia enfocados no exclusivamente a proporcionar una titulación que las permita acceder a un puesto de trabajo con el reconocimiento de expertas, que indiscutiblemente también es así, sino que para muchas suponen, además, un instrumento aprovechable para su enriquecimiento cultural. Las mujeres gaditanas en este tiempo no tienen la posibilidad de acceder a estudios superiores de secundaria, ni mucho menos universitarios, por lo que la Escuela Normal les da la posibilidad de formarse aunque no tengan la necesidad de ejercer como maestras en el futuro. Este argumento nos explica la demanda de este primer curso, que, aunque con cierto receso en el segundo y con un desplome en el curso 1860/61, se mantiene en el resto de la centuria.

En el caso de la Normal de maestros, podemos apreciar desde el curso 1857/58 hasta el 1880/81 un incremento progresivo que va desde los 14 alumnos del primer curso hasta los 82 del último año señalado. Conviene ahora señalar que en el transcurso de estos se contempla una matrícula de carácter oficial pero no hay constancia de ninguna matrícula libre hasta el curso 1868/69. La tendencia al alza se mantiene hasta alcanzar un número de alumnos matriculados que roza la centena en los cursos 1884/85 y 1888/89. La circunstancia más destacable es la posibilidad de cursar los estudios de magisterio a través de una matrícula libre establecida por el decreto de 21 de octubre de 1868 cuya entrada en vigor se refleja en el caso 
gaditano inmediatamente. A partir del curso 1890/91 y hasta finales del siglo, se inicia un descenso paulatino, seguramente debido al atractivo de otras ofertas educativas para los hombres y probablemente por la propia feminización que experimenta el ejercicio profesional del magisterio, si bien también en este caso se apunta una reducción de matrícula que podría estar en relación con otras posibilidades formativas abiertas también para las gaditanas, como es, según Flecha (1998, pp. 166-167), su incorporación paulatina a los institutos de segunda enseñanza.

Es sorprendente el pico que alcanza la matrícula en la Escuela Normal femenina en el curso 1885/86 con 157 alumnas, cifra que es superada en años posteriores y que se explica por la entrada en escena de una nueva forma de obtención del título, la enseñanza privada.

En general, en el último cuarto de siglo la matrícula en la Escuela Normal femenina es superior a la masculina. La diferencia radica en que, mientras que para los jóvenes la tendencia al descenso es una constante, en el caso de ellas se observa una fluctuación en los datos de matrícula, pues, aunque en casi todos estos años se mantiene por encima de las 100 alumnas, inicia un retroceso al finalizar el período estudiado.

\section{CONCLUSIONES}

Las Escuelas Normales influyeron de forma decisiva en la formación de los futuros decentes de la primera etapa educativa en Cádiz, asistiendo a formar un modelo de maestros y de maestras que, aunque en sus principios va cargado de aspectos segregadores por venir el currículum impuesto desde la propia normativa legal en la que se asienta, ofrece la posibilidad a las jóvenes de acceder a centros de instrucción superior para los que tradicionalmente encontraban sus puertas cerradas. Probablemente, este afán femenino de formación es lo que explica el nivel constante en la matrícula de ellas a lo largo del XIX.

En Cádiz, las mencionadas escuelas, aun siendo de las primeras en el territorio español, entra en escena bien avanzados los años cincuenta, coincidiendo con la proliferación de centros públicos en la ciudad, principalmente en el caso de a las niñas sin un personal que tenga la preparación adecuada, pues la preocupación por su formación había sido relegada a un segundo orden a favor de la masculina.

En general, estos centros de formación del profesorado, tanto la Normal masculina como la femenina, supusieron una mejora de la formación de los niños y de las niñas gaditanas, pues permitió proporcionar la adecuada instrucción intelectual y pedagógica a los aspirantes a maestros y maestras que se encargarían de la formación de la población infantil.

\section{REFERENCIAS BIBLIOGRÁFICAS}

- CAPel Martínez, R. Ma $\mathrm{M}^{\mathrm{a}}$ 1986. El trabajo y la educación de la mujer en España. Madrid: Instituto de la Mujer. 
- Capitán DíAZ, A., 1991. Historia de la Educación en España I. De los orígenes al Reglamento General de Instrucción Pública (1821). Madrid: Dykinson.

- GuZMÁn, M. de, 1986 Vida y muerte de las Escuelas Normales. Historia de la formación del Magisterio básico, Barcelona: PPU.

- Flecha Garcia, C., 1998. "La incorporación de las mujeres a los institutos de segunda enseñanza en España". En: Historia de la educación: Revista interuniversitaria, $\mathrm{n}^{\mathrm{o}} 17$ (1998), pp. 159-178.

- LoRenzo Vicente, J. A., 1995. "Perspectiva histórica de la formación del maestro en España (1370-1990)”. En: Revista Complutense de educación, vol. 6, nº 2 (1995), pp. 203229.

- Melcón Beltrán, J., 1992. La formación del profesorado en España (1837- 1914), Madrid: Ministerio de Educación y Ciencias.

- PÉRez Serrano, M., 1990. Las prácticas de enseñanza. Visión histórico-legislativa, Madrid: Universidad Autónoma.

- Llaza del Prado, A. y Pascual Pascual, Ma S., 2000. Los archivos de las Escuelas Normales de Cádiz. Siglo XIX. Cádiz: Servicio de Publicaciones de la Universidad.

- SCAnlon, G. M., 1987. "La mujer y la instrucción pública: de la Ley Moyano a la II República", en Historia de la Educación: Revista interuniversitaria, nº 6 (1987). Pp. 193207.

- VÁzQuez DomíngueZ, C. "Las escuelas normales del siglo XIX: la formación del profesorado en Cádiz" en Tavira: Revista de ciencias de la educación, no 26 (2010), pp25-53.

- Ventajas Dote, F. "La congregación de San Casiano y el ejercicio del magisterio de primeras letras en la Málaga setecentista”. Isla de Arriarán XXX, diciembre, 2007, pp. 4364. 